\title{
Cartographies moléculaires ultrastructurales par microscopie ionique
}

La microscopie ionique, méthode conçue et développée à l'université d'Orsay en France, permet une analyse ultrastructurale de la distribution d'éléments chimiques et de molécules, notamment dans le matériel biologique. Les renseignements qu'elle permet d'obtenir sont du même type que ceux apportés par la microautoradiographie, mais sa sensibilité et sa rapidité d'exécution pourraient permettre d'aborder des sujets autrefois inaccessibles, notamment dans le domaine de la pharmacologie cellulaire. Le principe de cette méthode est de bombarder la surface à analyser avec des ions primaires. Les ions secondaires arrachés à la matière par ce bombardement sont alors triés par un spectromètre de masse. Les images obtenues représentent donc la distribution des atomes étudiés dans le volume analysé.

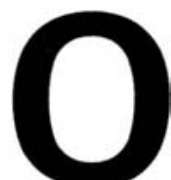

btenir en quelques secondes ou minutes des images ultrastructurales représentant les distributions des différents éléments chimiques ou molécules présents à l'état de trace au sein des cellules aurait semblé irréaliste il y a peu de temps encore. C'est pourtant aujourd'hui possible, grâce au développement récent de la microscopie ionique, une méthode conçue et mise au point à l'université d'Orsay [1]. Ces développements faits à l'ONERA* ont permis de franchir une étape décisive : le nouvel instrument réalisé en 1990 [2] permet

P. Galle : membre correspondant de l'Académie toire de biophysique. SC 27 de l'Inserm, faculté de médecine, 8, rue du Général* Office national d'études et de recherches aérospa-
tiales, Châtillon-sous-Bagneux, France. d'obtenir maintenant des cartographies chimiques ultrastructurales d'éléments ou de molécules présentes en très faible concentration. Ces caractéristiques présentent un intérêt particulier dans le domaine de la recherche biologique.

La microscopie ionique s'ajoute donc aujourd'hui à l'arsenal des méthodes classiques de microlocalisation intracellulaire - comme la cytochimie, l'immunocytochimie ou la microautoradiographie - , mais cette nouvelle méthode a, sur ces dernières, de nombreux avantages, et notamment sa simplicité, sa rapidité d'exécution et sa spécificité. En outre et surtout, son domaine d'application, beaucoup plus vaste, permet d'aborder des sujets autrefois inaccessibles. 
C'est dans les domaines de la biologie cellulaire, de la biologie moléculaire et de l'étude des sites récepteurs des médicaments, hormones ou enzymes, que les nouveaux microscopes ioniques devraient trouver leurs applications les plus immédiates. Nous présentons ici quelquesunes de ces applications.

Avant de montrer ses possibilités actuelles dans le domaine de la recherche biomédicale, un bref rappel des principes de cette méthode, encore peu connue des biologistes, est nécessaire.

\section{Principe des microscopes ioniques}

En 1960, R. Castaing et G. Slodzian proposaient une nouvelle méthode de microanalyse locale chimique et isotopique utilisant le phénomène d' "émission ionique secondaire " [1]. Cette méthode, largement utilisée actuellement pour l'étude des matériaux inertes, s'applique tout aussi bien à celle des structures biologiques [3]. La surface d'un échantillon solide (cellules étalées, coupes de tissu) est bombardée par un faisceau d'ions (ions primaires). Sous l'influence de ce bombardement, les atomes de la surface de l'échantillon sont progressivement arrachés, notamment sous forme ionisée (ions secondaires)* ${ }^{*}$ Ces ions secondaires, caractéristiques d'une région donnée de la surface de l'échantillon, sont collectés, accélérés par une différence de potentiel et triés par un spectromètre de masse. L'image, représentant la distribution des atomes ainsi sélectionnés dans une région donnée de la préparation, peut être obtenue par microscopie directe ou en utilisant un dispositif de microscopie à balayage.

Dans un microscope ionique, l'image est donc formée avec les propres atomes de l'échantillon, et le système d'analyse étant un spectromètre de masse, il est possible de distinguer entre eux les différents isotopes, naturels ou artificiels, des éléments présents.

\footnotetext{
- Selon la nature de l'atome, les ions secondaires sont émis sous forme positive ou sous forme négative. La plupart de ces ions ne portent qu'une seule charge. Un faible pourcentage d'entre eux peut porter deux ou plusieurs charges.
}

- Le microscope ionique à image direct (figure 1).

La préparation est bombardée par les ions primaires sur l'ensemble de la surface à étudier, par exemple par un faisceau de $300 \mu \mathrm{m}$ de diamètre. L'échantillon est érodé au rythme d'environ une couche atomique par seconde. Les ions secondaires émis de la préparation sont accélérés par une différence de potentiel (positive ou négative selon la variété d'ions que l'on désire collecter), puis focalisés en un faisceau par une optique électrostatique. Ce faisceau transporte en chaque instant l'image globale de tous les ions émis. Ce faisceau

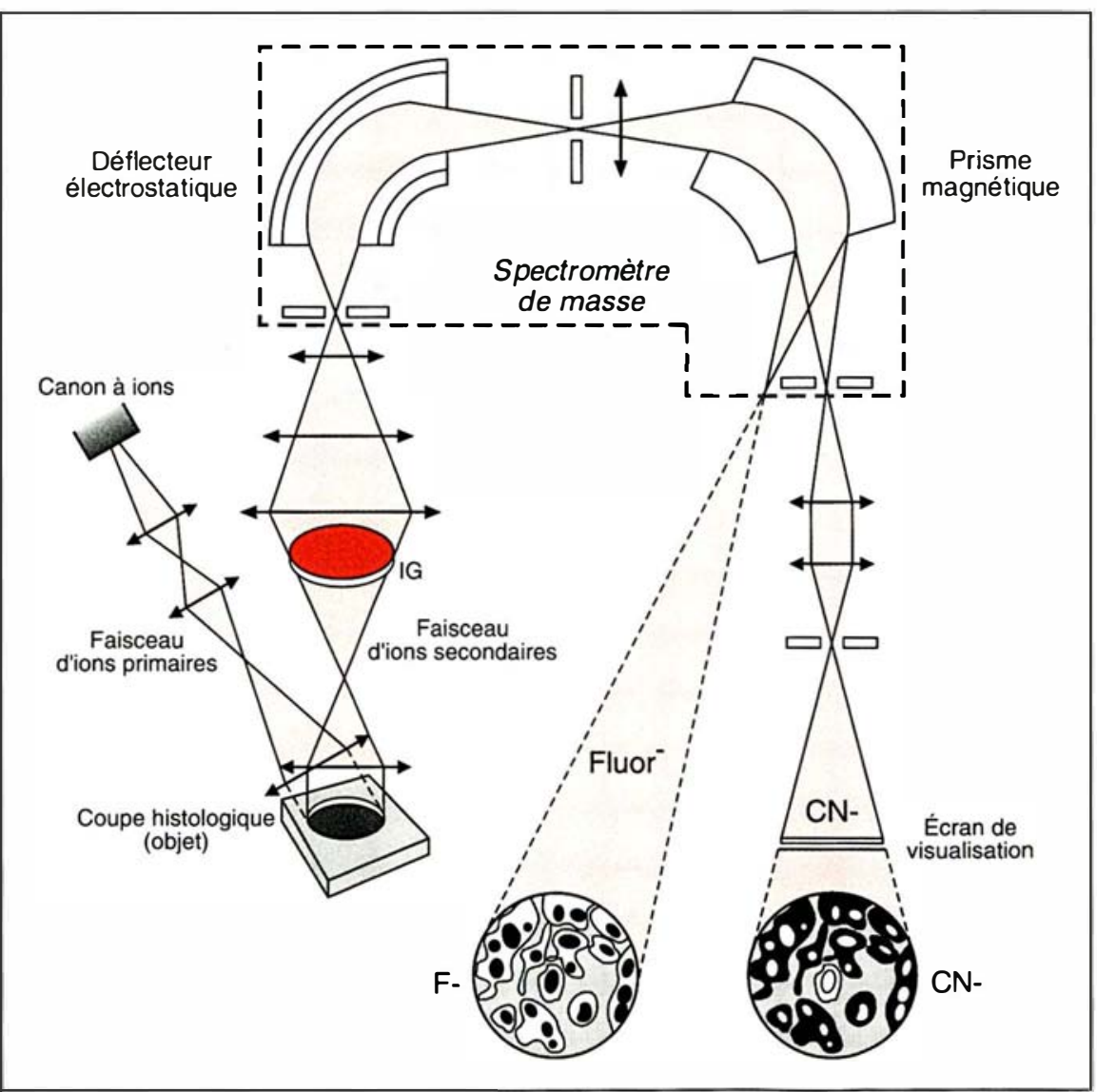

Figure 1. Schéma d'un microscope ionique à image directe. Le faisceau d'ions secondaires émis de la préparation sous l'impact des ions primaires transporte l'image globale IG de tous les atomes émis sous forme d'ions négatifs. Ces atomes sont triés par le spectromètre de masse à la sortie duquel on peut sélectionner le seul faisceau transportant la variété d'atome que l'on désire imager. L'image de la répartition de cet atome, ici l'atome d'azote émis de la cellule sous forme d'ions ${ }^{26} \mathrm{CN}^{-}$apparaît directement sur l'écran. L'image de la répartition dans cette même cellule de l'atome de fluor serait obtenue en sélectionnant le faisceau transportant les ions de masse $19\left(^{19} \mathrm{~F}^{-}\right)$, cette image est représentative de la distribution d'une molécule fluorée, ici la trifluoropromazine, qui s'incorpore exclusivement dans le cytoplasme. 
l'image analytique représentant la distribution de l'ion sélectionné apparaît directement sur l'écran fluorescent ; elle peut être aussi fixée sur un film photographique. En faisant varier le champ magnétique du spectromètre, on obtient successivement sur l'écran les images analytiques des différents éléments ou isotopes présents. Les images obtenues par cette première méthode ont une résolution de l'ordre de $0,5 \mu \mathrm{m}$.

- Le microscope ionique à balayage Cet instument permet d'obtenir des images de résolution ultrastructurale. La préparation est ici bombardée par une sonde ionique hyperfine dont le diamètre est de quelques dizaines de nanomètres. Sous l'impact de cette sonde, un très petit volume de matière est pulvérisé et les atomes ainsi émis sont triés par le spectromètre de masse. Le signal ainsi obtenu, proportionnel au nombre d'atomes de la variété sélectionnée, est adressé sur l'écran d'un oscillographe cathodique pour former un pixel* de l'image. Le balayage point par point de la sonde ionique sur la surface de la préparation, synchronisé avec celui de l'oscillographe cathodique, permet d'obtenir la cartographie représentant la distribution de la variété atomique recherchée. Dans cette image digitale, chaque point image (pixel) peut être mis en mémoire et le nombre d'atomes spécifiques émis de chaque volume successivement pulvérisé (voxel) peut être compté.

L'appareil que nous avons émis a été réalisé par G. Slodzian et al. [2] à l'ONERA. La sonde ionique est constituée d'ions Césium. L'intensité de la sonde est de l'ordre de 6 millions d'ions par seconde. Le temps d'impact en chaque point est de l'ordre de 30 millisecondes, provoquant l'érosion d'environ 5 couches atomiques. Le microvolume pulvérisé est donc approximativement un cylindre de diamètre égal à celui de la microsonde et de $2 \mathrm{~nm}$ de hauteur. Le nombre d'atomes ainsi éjectés par impact est de l'ordre de 500000.

Parmi les 500000 atomes pulvérisés, Ion microscopy: a new approach for subcellular localization of labelled molecules Scanning Microscopy 1988; 2 : 1821-9.

6. Berry JP, Lespinats G, Escaig F, Boumati $P$, Tlouzeau S, Cavellier JF. Intracellular localization of drugs in cultured tumor cells by ion microscopy and image proces-
* Un pixel est un élément constitutif d'une image digitale. Il définit un point de cette image. Une image de $256 \times 256$ pixels est ainsi formée de 65536 points image. quatre variétés peuvent être sélectionnées simultanément, permettant l'obtention de quatre images analytiques différentes pour chaque exploration de la surface imagée.

Les surfaces dont l'image est analysée sont des carrés dont les dimensions varient actuellement de $5 \times 5 \mu \mathrm{m}$ à $20 \times 20 \mu \mathrm{m}$. La résolution obtenue est fixée par la dimension de la sonde. Le nombre d'impacts utilisés par balayage définit le nombre de pixels de l'image (généralement $256 \times 256$ ou $512 \times 512$ points images) .

\section{Cartographies moléculaires par microscopie ionique}

Les microscopes ioniques sont généralement utilisés pour faire des images élémentaires ou isotopiques, mais ils permettent également d'obtenir des images représentant la distribution des molécules présentes [4-6]. Ces cartographies moléculaires peuvent être obtenues directement pour toute molécule contenant dans leur structure un atome permettant de les reconnaître (fluor, iode, brome, platine), ce qui est le cas de nombreuses drogues pharmaceutiques (corticoïdes, benzodiazépines, anticancéreux) ou d'analogues pyrimidiques. Dans ces cas, l'image représentant la distribution de l'atome spécifique est également représentative de la distribution de la molécule, dans la mesure où celle-ci n'a pas été catabolisée.

S'agissant des autres molécules, il est nécessaire préalablement de les marquer par un isotope qui peut être indifféremment stable (deutérium, carbone 13 , azote 15 , oxygène 17 ) ou radioactif (carbone 14), puisque la détection est ici faite par spectrométrie de masse.

La microscopie ionique présente plusieurs avantages importants sur la microautoradiographie : (1) les images sont obtenues en quelques secondes ou minutes au lieu de quelques jours ou mois ; (2) la résolution spatiale est améliorée d'un facteur 10 ; (3) plusieurs molécules, marquées chacune par un isotope différent, peuvent être étudiées au sein d'une même cellule et leur répartition comparée ; (4) la sensibilité de détection est généralement meilleure, notam- 
ment pour le carbone 14 ; (5) les appréciations quantitatives sont plus simples ; et (6) la possibilité d'utiliser les isotopes stables au lieu d'isotopes radioactifs évite les phénomènes de radiolyse; elle permet en outre d'envisager des études in vivo chez l'homme. En revanche, il n'est pas encore possible en microscopie ionique, comme c'est le cas en microautoradiographie, d'observer simultanément la préparation en microscopie électronique classique.

\section{Possibilités offertes par la microscopie ionique en imagerie moléculaire intracellulaire}

- Microscopie ionique à image directe (basse résolution)

La figure 2 montre deux images analytiques obtenues sur la même plage d'une préparation de cellules nerveuses en culture (souche C6 d'un astrocytome humain) après incubation pendant 24 heures dans un milieu contenant une phénothiazine (trifluoropromazine). La préparation est ici un étalement cellulaire en monocouche. L'image de droite montre la répartition intracellulaire de la trifluoropromazine. Cette image est obtenue par utilisation des atomes de fluor émis des molécules sous forme d'ions secondaires négatifs de masse $19\left({ }^{19} \mathrm{~F}\right)$. La molécule est distribuée de façon à peu près homogène dans le cytoplasme. Elle est absente du noyau. A cette résolution, il n'est pas possible de distinguer les organites intracytoplasmiques.

La figure 3 (p. 948) montre la distribution d'un corticoïde, la dexaméthazone, au sein de fibroblastes humains en culture après 24 heures d'incubation en présence de cette molécule. On voit qu'ici la distribution de la drogue est intranucléaire. Au sein des noyaux, la distribution est à peu près homogène sauf en certains points correspondant sans doute aux nucléoles. Cette image montre aussi la limite de résolution des microscopes ioniques à image directe, insuffisante ici pour établir un rapport entre la localisation intranucléaire de cette molécule et celle de l'acide désoxyribonucléique.

- Microscopie ionique à balayage (haute résolution)

- Incorporation de trois molécules, analogues pyrimidiques, dans des cellules cancéreuses en culture.

Les cellules proviennent d'un cancer mammaire humain (souche MCF7). A près incubation pendant 24 heures dans un milieu contenant à la fois de la bromodéoxyuridine, de l'iododéoxyuridine et du fluoro-

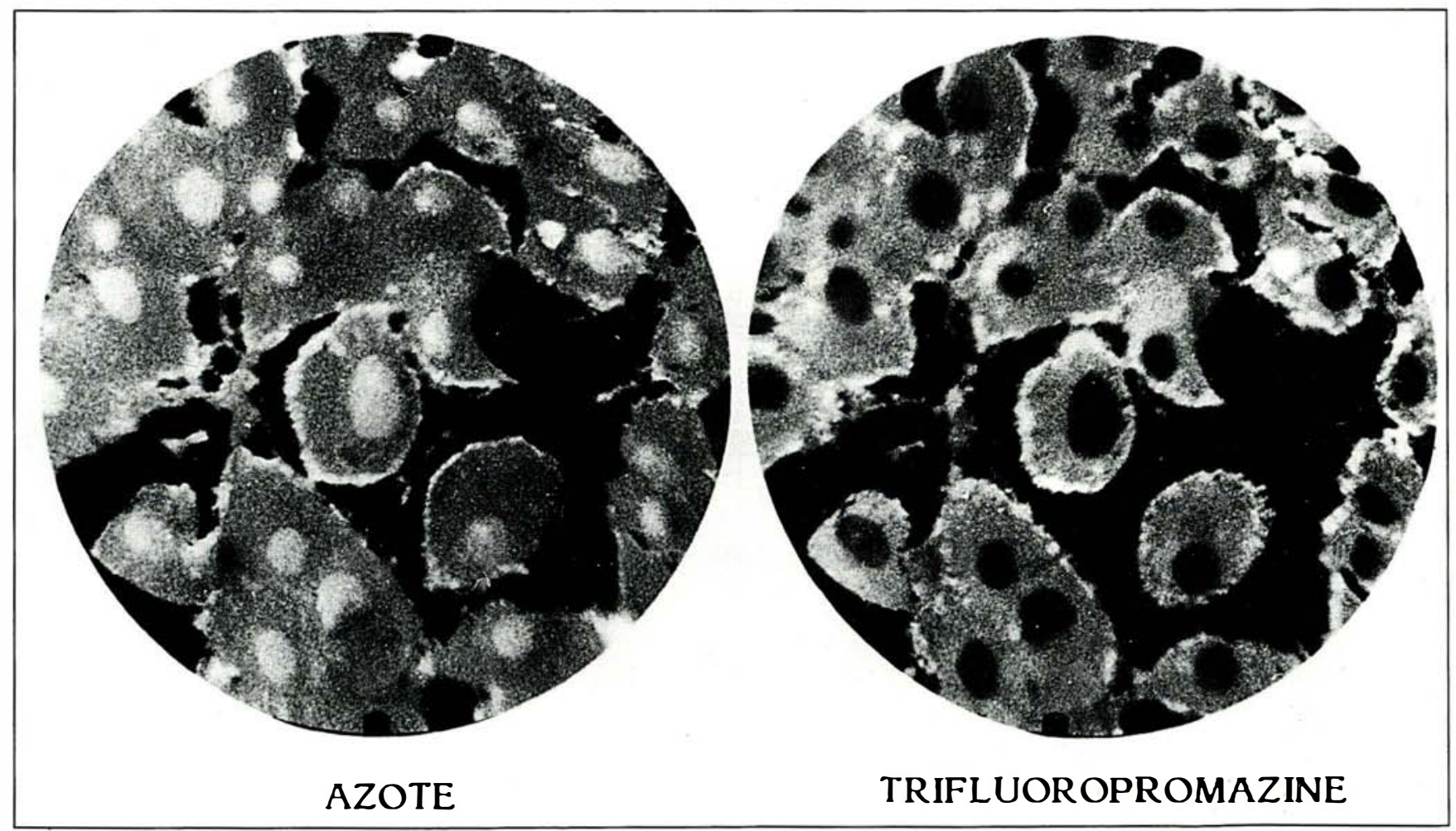

Figure 2. Microscopie ionique à image directe (basse résolution). Cellules d'une souche d'un astrocytome humain (souche C6) cultivées en monocouche et incubées 24 heures dans un milieu contenant de la trifluoropromazine. A gauche : image de la distribution de l'azote permettant de distinguer l'emplacement du cytoplasme et celui des noyaux. A droite : image de la distribution du fluor dans ces même cellules. Cette image est représentative de la distribution intracellulaire de la molécule fluorée. Cette molécule est distribuée de façon homogène dans le seul cytoplasme. (Grossissement : $X$ 400.) 
uracile, les cellules ont été fixées et incluses dans l'épon. Les images de la distribution des trois molécules incorporées ont été obtenues sur des coupes d'épon de $1 \mu \mathrm{m}$ d'épaisseur. La figure 4 montre cinq images analytiques obtenues sur l'une de ces cellules. Les cinq variétés d'atomes dont l'image est étudiée sont le soufre, le phosphore, le brome, l'iode et le fluor. L'image soufre est grossièrement représentative de la distribution des protéines; et leur intensité, de leur richesse en soufre. L'image phosphore est essentiellement représentative de la distribution des acides nucléiques. Les trois images suivantes sont spécifiques des trois molécules incorporées :

- les images brome et iode sont représentatives de la distribution de la bromodéoxyuridine et de l'iododéoxyuridine. Ces deux molécules sont des analogues de la thymidine et s'incorporent spécifiquement dans l'ADN. Ces deux images sont donc aussi représentatives de la distribution de l'acide désoxyribonucléique ayant incorporé ces analogues pyrimidiques pendant le temps d'incubation. On remarque l'absence d'incorporation dans les deux nucléoles et un renforcement de l'émission autour de ces deux nucléoles. La répartition dans le noyau de ces deux analogues pyrimidiques est identique, mais la concentration de la bromodéoxyuridine est plus élevée que celle de l'iododéoxyuridine, traduisant le fait que, lorsque l'incorporation de ces deux molécules est faite simultanément, l'incorporation de la bromodéoxyuridine est prédominante ;

- l'image fluor est représentative de la distribution du fluoro-uracile, une molécule s'incorporant plus spécifiquement dans l'ARN. Cette molécule est présente dans les deux nucléoles ;

- chacune des images précédentes a été obtenue par pulvérisation de quelques couches atomiques de la préparation, sans doute moins de 10 couches, correspondant à une épaisseur de la coupe d'épon de l'ordre de $3 \mathrm{~nm}$ seulement.

La figure 5, obtenue sur une autre cellule de la même préparation,

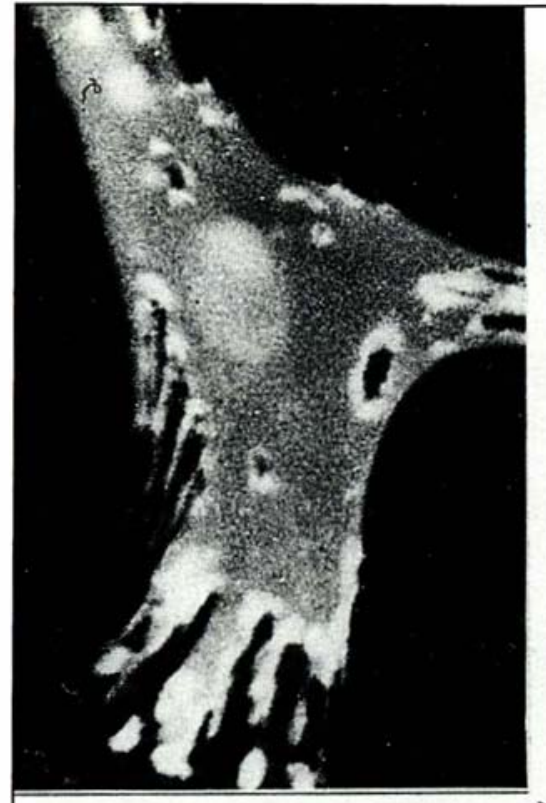

AZOTE

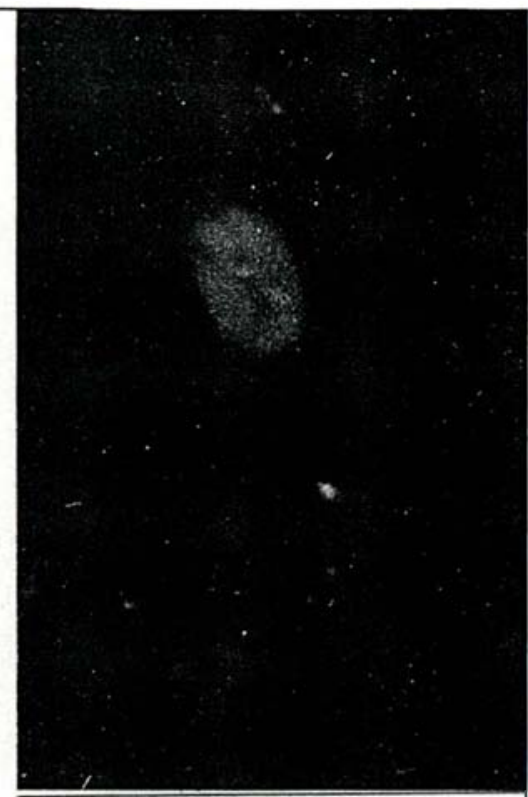

FLUOR

(DEXAMETHAZONE)

Figure 3. Microscopie ionique à image directe (basse résolution). Fibroblaste humain en culture incubé 24 heures dans un milieu contenant de la dexaméthazone. A gauche : image de la distribution de l'azote permettant de distinguer l'emplacement du noyau de celui du cytoplasme. A droite : image de la distribution du fluor, représentative de la distribution de ce corticoïde fluoré. La molécule est concentrée dans le noyau dans lequel sa distribution n'est pas parfaitement homogène. Les points noirs correspondent probablement aux nucléoles n'ayant pas incorporé la molécule. (Grossissement : $X$ 500.)

la bromodéoxyuridine. Il est intéressant de comparer cette image à celles représentant les distributions des éléments soufre, azote et phosphore.

- l'image brome, spécifique de la distribution de l'ADN synthétisé pendant l'incubation, apparaît sous forme de plusieurs taches très brillantes : la cellule est en métaphase et chacune de ces taches correspond à l'emplacement d'un chromosome ; 18 chromosomes sont ainsi individualisés dans cette région de la coupe correspondant à la pulvérisation d'environ 10 couches atomiques ;

- sur l'image phosphore, les chromosomes sont également bien individualisés et deux zones supplémentaires, moins riches en phosphore, sont également mises en évidence (flèches); ces zones peuvent être interprétées comme correspondantes à l'emplacement de deux nucléoles ; - sur l'image azote, les chromo- somes et nucléoles, tous deux riches en cet élément, sont également bien visibles au sein du noyau. Le nucléoplasme et le cytoplasme apparaissent en outre avec, dans ce dernier, les organites intracytoplasmiques riches en azote (mitochondries, lysosomes). La membrane nucléaire n'est pas individualisée comme elle le serait si la cellule n'était pas en métaphase ;

- sur l'image soufre, les chromosomes ne sont pas visibles, témoignant du fait que leur teneur en soufre n'est que très peu différente de celle du reste du nucléoplasme. Les deux zones interprétées comme étant des nucléoles (flèches) sont également riches en soufre, de même que les organites intracytoplasmiques individualisés sur l'image azote. Aucune membrane ne sépare le cytoplasme du hyaloplasme dans cette cellule en métaphase.

- Cartographie ultrastructurale d'une 


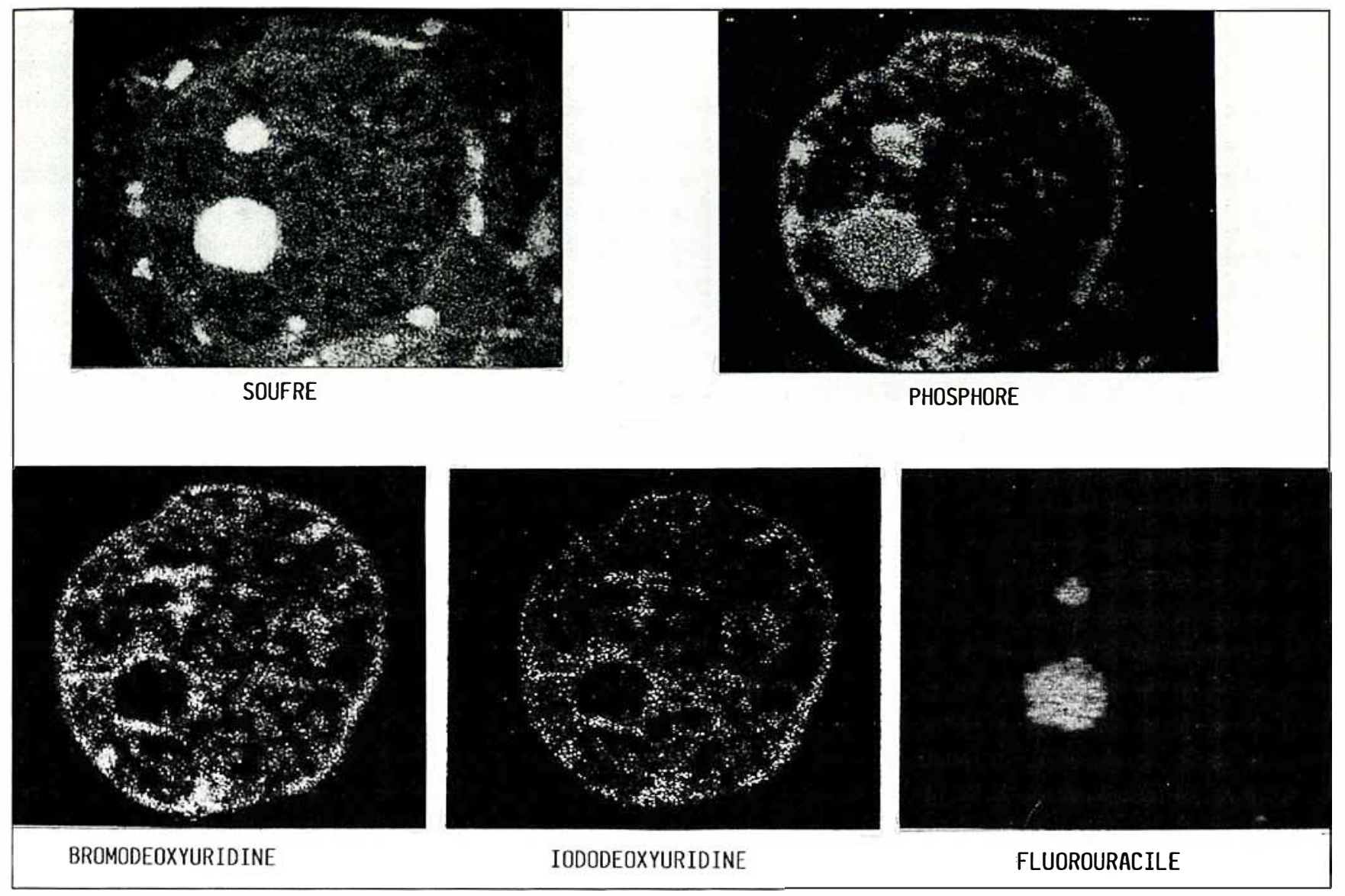

Figure 4. Microscopie ionique à balayage (haute résolution). Cinq images analytiques obtenues sur une même cellule d'un carcinome mammaire humain en culture (souche MCF-7). Trois molécules différentes ont été introduites 24 heures dans le milieu de culture (bromodéoxyuridine, iododéoxyuridine et fluoro-uracile). L'image soufre permet de reconnaître les structures cellulaires. Dans le noyau, les deux zones brillantes correspondent à l'emplacement de deux nucléoles très riches en soufre (ribonucléoprotéines). Dans le cytoplasme, la plupart des organites très émissifs correspondent aux mitochondries et les zones noires sont des vacuoles ou des inclusions lipidiques. La membrane nucléaire est individualisée. L'image phosphore est représentative de la distribution des molécules phosphorées non diffusibles, principalement des acides nucléiques (les petites molécules phosphorées ont été éliminées lors de la fixation). Dans le noyau, on distingue l'hétérochromatine et les deux nucléoles. On observe un renforcement de la concentration en phosphore autour des deux nucléoles. Les images de la bromodéoxyuridine et de l'iododéoxyuridine sont obtenues respectivement par sélection des ions brome et iode. Ces images sont représentatives de la distribution de l'ADN ayant incorporé ces molécules pendant la période d'incubation. On remarque les mottes d'hétérochromatine en périphérie du noyau, et un renforcement autour des deux nucléoles. L'image fluor, représentative de la distribution du fluoro-uracile montre que cette molécule s'est incorporée spécifiquement dans l'ARN des deux nucléoles.

molécule marquée par un isotope stable. Lorsqu'une molécule ne contient pas dans sa structure un atome spécifique permettant de la reconnaître, il est nécessaire de la marquer, et le marqueur peut être un isotope stable.

Les cellules de la souche précédente (MCF-7) ont été mises en culture et incubées dans un milieu contenant de l'adénine marquée avec de l'azote 15.

$m / s n^{\circ} 9$, vol. 7 , novembre 91
Quatre images analytiques sont présentées sur la figure 6 ( $p$. 951). L'image soufre met en évidence trois nucléoles au sein du noyau cellulaire. L'un de ces nucléoles est plus riche en soufre que les deux autres. Sur l'image azote, on retrouve les trois nucléoles dont les concentrations relatives en cet élément ne sont pas les mêmes que celles du soufre. Sur l'image phosphore, deux nucléoles seulement sont très riches en cet élement. L'adénine est une base qui s'incorpore aussi bien dans l'ADN que dans l'ARN. L'image représentant sa distribution témoigne donc de la présence de ces deux molécules. Dans ce noyau, on constate que l'adénine N15 est présente dans l'ensemble du nucléoplasme où elle apparaît sous forme de petites mottes disséminées. S'agissant des nucléoles, un seul parmi les trois a 
fortement incorporé l'adénine N15, celui dont la teneur en soufre est la plus élevée témoignant du fait que, durant la phase d'incubation, les deux autres nucléoles n'ont pas synthétisé d'ARN. Ainsi est mise en évidence ici une hétérogénéité de fonctionnement de différents nucléoles au sein d'un même noyau.

\section{Les limites de l'analyse chimique localisée}

En microscopie ionique, l'identification d'un atome présent en une région donnée de l'échantillon se fait par spectrométrie de masse, une méthode dans laquelle le "bruit de fond " est extrêmement faible. Il en résulte que les limites ultimes de l'analyse chimique peuvent être approchées d'assez près.

Un seul atome, émis sous forme ionisée d'une zone submicroscopique de l'échantillon, et arrivant jusqu'au détecteur, peut donner lieu à un signal (point lumineux sur l'écran). Un atome unique présent dans le microvolume pulvérisé pourrait donc en principe être ainsi, à la fois, détecté, identifié et localisé avec une précision submicroscopique. Cette limite ultime n'est cependant pas atteinte car un certain nombre d'atomes sont perdus pour l'analyse, et notamment ceux qui ne sont pas émis sous une forme ionisée (les atomes neutres ne peuvent pas être collectés) et ceux arrêtés par le diaphragme ou fentes du spectromètre. G. Slodzian a montré, par exemple [2], qu'il faut pulvériser au moins 200 atomes de carbone pour obtenir un signal analytique $\mathrm{C}$ - . Le " rendement utile " défini comme le rapport entre le nombre d'ions détectés sur le nombre d'atomes du même élément qu'il est nécessaire de pulvériser du même volume pour obtenir un signal est ici de 1/200. S'agissant des autres éléments, ce rapport peut être plus élevé ou plus faible, dépendant des caractéristiques physicochimiques de l'élément et notamment de sa probabilité d'être émis sous forme d'une particule chargée.

En microanalyse, la sensibilité peut s'exprimer en masse minimale détectable ou en concentration minimale

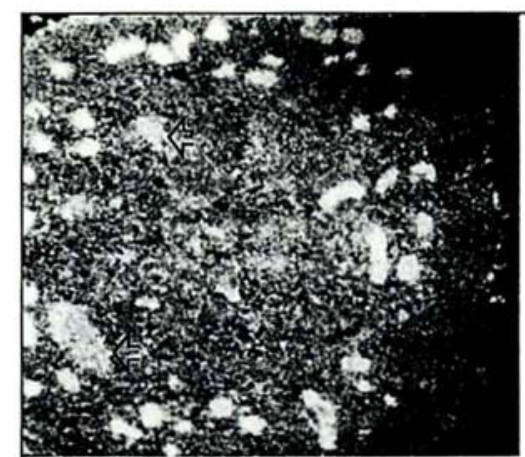

SOUFRE

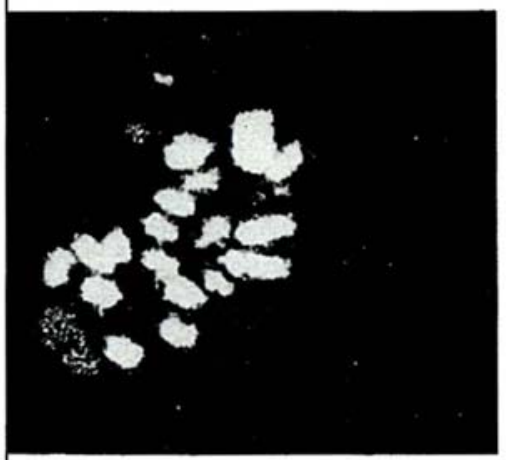

PHOSPHORE

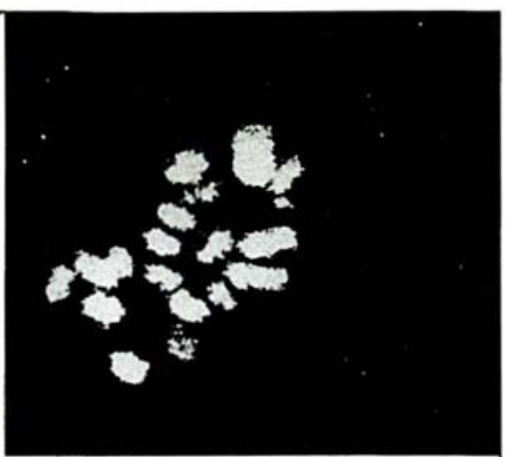

BROME

Bromodeoxyuridine

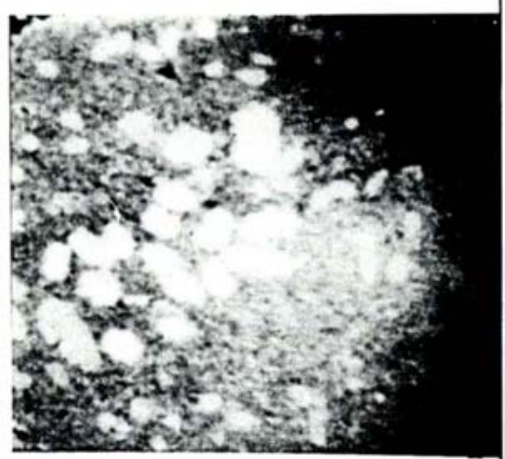

AZOTE

Figure 5. Même préparation que la figure 4. La phase du cycle cellulaire est ici différente. Sur l'image soufre, le noyau n'est pas individualisé. La membrane nucléaire n'est pas visible. Deux zones plus brillantes sont indiquées par des flèches. En périphérie, on observe les organites intracytoplasmiques. La distribution de la bromodéoxyuridine est très différente de celle observée sur la figure 4. On observe dans le noyau 18 taches très brillantes correspondant aux chromosomes. La cellule est en métaphase. Sur l'image phosphore, on observe, outre les 18 chromosomes, deux autres zones correspondant à celles individualisées sur l'image soufre par les deux flèches. Sur I'image azote, on retrouve les chromosomes, les organites intracytoplasmiques et les deux zones individualisées sur les images soufre et phosphore. Ces deux zones peuvent être interprétées comme étant le reste de deux nucléoles.

minimale détectable est celle de 200 atomes (moins de $10^{-20} \mathrm{~g}$ ). La concentration minimale détectable est une notion souvent plus utile en biologie, elle dépend de la dimension du volume pulvérisé. Sous une sonde de $100 \mathrm{~nm}$ de diamètre pulvérisant 10 couches atomiques, soit un volume contenant environ 1 million d'atomes, la concentration minimale détectable est $200 / 10^{6}=2.10^{-4}$ en concentration atomique pour une résolution de $100 \mathrm{~nm}$. Si ces 200 atomes de carbone étaient répar- tis dans un volume 200 fois plus grand (un cube de 0,3 $\mu \mathrm{m}$ de côté), la concentration minimale détectable serait de l'ordre de $1 \mathrm{ppm}$.

Sur le plan pratique, il est possible d'obtenir des images de la majorité des éléments présents à une concentration de $1 \mathrm{ppm}$ dans de tels volumes. Dans les milieux biologiques, les molécules agissent généralement à très faible concentration et cette très haute sensibilité est souvent nécessaire.

Il est utile de comparer cette sensi- 


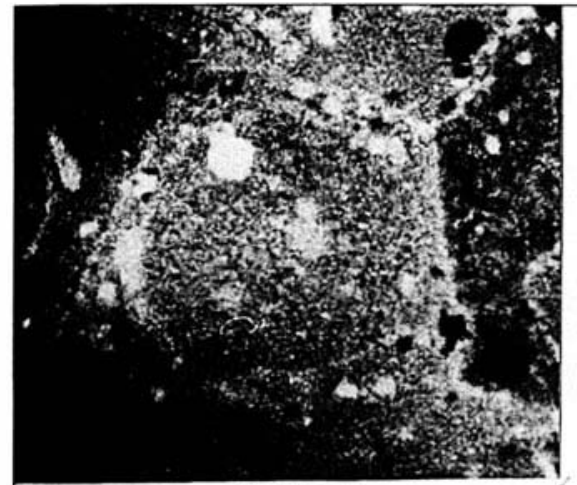

SOUFRE

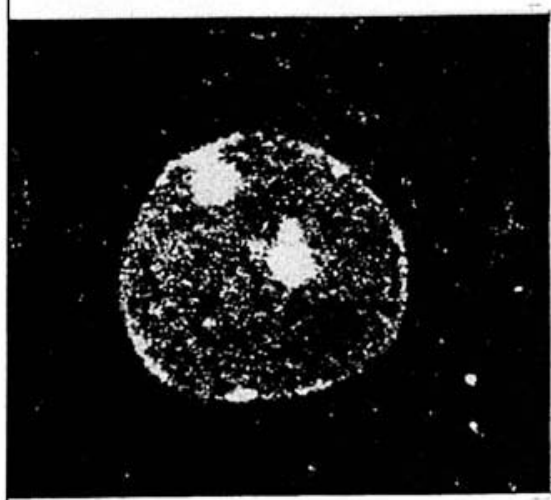

PHOSPHORE

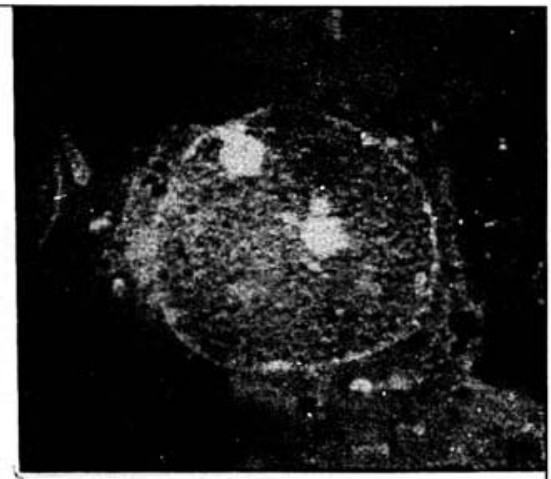

AZOTE 14

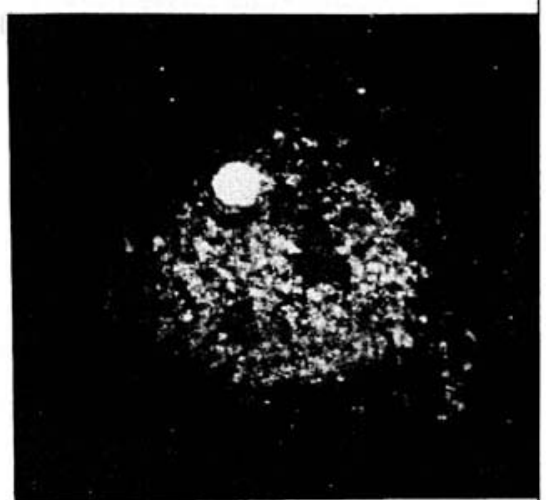

AZOTE 15 (ADEN NE N15)

Figure 6. Exemple de l'utilisation d'un isotope stable. De l'adénine marquée par l'azote 15 a été ajoutée au milieu de culture des cellules de la souche MCF-7. Cette molécule s'incorpore à la fois dans I'ADN et dans I'ARN. Sur l'image soufre on observe trois nucléoles dans le noyau, dont l'un seulement est très riche en soufre. Sur les images azote 14 et phosphore, deux de ces nucléoles sont également riches en ces deux éléments naturels. L'image de l'azote 15, représentative de la distribution de l'adénine marquée, montre que l'adénine s'est préférentiellement incorporée dans un seul des trois nucléoles, celui riche en soufre. L'adénine est également présente dans le reste du nucléoplasme; elle est pratiquement absente dans les deux autres nucléoles.

bilité avec celle de la microautoradiographie, une autre méthode de microlocalisation intracellulaire réputée pour sa très haute sensibilité. En microautoradiographie, un seul atome de carbone 14, émettant un rayonnement bêta, peut être visualisé par une trace sur une émulsion sensible, et ainsi localisé avec une précision de l'ordre de 0,2 $\mu \mathrm{m}$. Cependant, compte tenu de la période du carbone 14 (5 700 ans), la fraction d'atomes qui se désintègre en un temps donné est très faible, et on $m / s n^{\circ} 9$, vol. 7 , novembre 91 radioélément de période plus courte, comme le tritium (période de 12 ans), montrerait une sensibilité équivalente des deux méthodes. C'est uniquement pour des radioélements de période encore plus courte, comme l'iode 125 ( $\mathrm{T}=2$ mois), que la sensibilité de détection est meilleure par les méthodes radioactives que par celles utilisant la spectrométrie de masse.

\section{Conclusion}

La microscopie ionique est une méthode d'exploration analytique submicroscopique aux applications multiples. Cette méthode a deux caractéristiques qu'elle ne partage avec aucune autre: sa très haute sensibilité et sa capacité d'analyse isotopique. Ces deux caractéristiques permettent d'obtenir aisément des cartographies chimiques ultrastructurales représentant la distribution de tout élément ou isotope présent à l'état de trace dans les cellules, depuis les trois isotopes de l'hydrogène jusqu'à ceux des transuraniens. Plus intéressantes en biologie sont sans doute les possibilités offertes de cartographies moléculaires avec ou sans marquage.

De même que les physiciens s'intéressant à la structure des matériaux, les biologistes disposent maintenant d'un nouvel outil dont les principales applications devraient se situer dans les domaines de la cytochimie, de la pharmacologie cellulaire et de la biologie moléculaire

\section{Remerciements}

Nous tenons à remercier vivement la direction de l'Office national d'études et de recherches aérospatiales qui nous a permis d'utiliser le prototype de sonde ionique conçu et réalisé dans ses laboratoires dans le cadre d'une coopération entre l'ONERA (B. Daigne) et l'université Paris XI (G. Slodzian). Nous remercions aussi nos collègues biologistes pour l'aide précieuse apportée dans la préparation des échantillons, et notamment : Mmes Tardy et Zhang, MM. Berry, Uriel, Beaupin et Hindie, ainsi que Mme Escaig et M. Hillion pour leur grande compétence en microscopie ionique.

(Summary page suivante) 


\section{Summary}

Mapping the subcellular distribution of biological molecules by ion microscopy

Analytical ion microscopy, a method proposed and developped by R. Castaing and G. Slodzian in Orsay university (France) has been applied to the study of the subcellular distribution of biological molecules and pharmaceutical drugs. This method makes it possible to easily and rapidly obtain analytical images representing the distribution of elements or isotopes in a tissue section (from the three isotopes of hydrogen to transuraniens elements), even when these elements or isotopes are at a trace concentration (1 ppm or less). Recent developments allow to obtain these chemical images at the ultrastructural level. In this paper, images representing the subcellular distribution of halogeneous molecules (pharmaceutical drugs, pyrimidic analogues) and of molecules labeled with a stable isotope are presented.

\section{TIRÉS A PART}

P. Galle. 